\title{
Collagen distribution in the lamina cribrosa and the trabecular meshwork of the human eye
}

\author{
MATS REHNBERG,' THORKIL AMMITZBÖLL, ' AND BJÖRN TENGROTH' \\ From the 'Department of Ophthalmology, Karolinska Institute and Hospital, S-104 01 Stockholm, Sweden, \\ and 'Department of Dermatology, Rigshospitalet, DK-2100 Copenhagen, Denmark
}

SUmmary Tengroth and Ammitzböll found the collagen content of the optic disc in glaucoma to differ from that of normal eyes. A theory was advanced that a primary collagen disturbance might be involved in the pathogenesis of glaucoma. The connective tissue in the body has a supportive function in almost all the organs. The tensile strength and elasticity of connective tissue is mainly due to the presence of collagen fibres and elastic fibres, which also maintain the shape of the tissues. There are many different types of collagen, three of which are discussed in this paper. Type I collagen is found in tendons, skin, and numerous other organs, for example the eye. Type III is found mainly in the blood vessels but is also present in other tissues with a mesodermal origin, and type IV is found in the basement membranes. To elucidate the precise distribution of collagen types in the ocular structures an immunhistochemical study was undertaken in normal human eyes. The amino acids proline, hydroxyproline, and hydroxylysine, which are characteristic of collagen, were also analysed. Collagen types I, III, and IV were found in the lamina cribrosa, the trabecular meshwork, and the retrolaminary optic nerve. In contrast, only type I was found in the sclera.

Two structures which are of particular importance in connection with chronic open angle glaucoma are the trabecular meshwork and the lamina cribrosa. The hypothesis has been advanced that abnormalities in the composition of collagen, which provides the supportive framework, might cause decreased pressure resistance in these tissues. The result might be cupping of the optic disc and closure of the trabecular mesh, which in turn would cause strangulation of the nerve fibres and an increased outflow resistance.

In a previous study' the amounts of the collagenspecific amino acids proline (Pro), hydroxyproline (Hyp), and hydroxylysine ( $\mathrm{Hyl}$ ) in the trabecular meshwork, the lamina cribrosa, and the sclera of glaucomatous and normal human eyes were analysed. Significantly increased concentrations of Hyp, Hyl, and Pro were found in the optic disc of glaucomatous eyes, but the concentrations of the three amino acids in the trabecular meshwork and the sclera were found to be unaltered.

In the same study the collagen amino acid relationship, as reflected by the Hyp to Hyl ratio, was found

Correspondence to Dr M Rehnberg. to be different in the trabecular meshwork, the optic disc, and the sclera. The lamina cribrosa, however, had not been separated from the retrolaminar tissue.

In the present study the lamina cribrosa has been isolated from the retrolaminar tissue, and the amino acid ratios of hydroxyproline (Hyp) and hydroxylysine (Hyl) have been determined for the trabecular meshwork, sclera, lamina cribrosa, and retrolaminary optic nerve. Differences between tissues in the ratios of these amino acids indicate that the tissues have different collagen compositions, although the amino acid ratios cannot be used to determine which types of collagen are present. Three types of collagen are of interest, namely, types I and III, which are interstitial collagens, and type IV, which is a basement collagen. We have therefore used antibodies to collagen types I, III, and IV with the peroxidase-antiperoxidase (PAP) technique to study the collagens immunohistochemically in the same four types of tissues that were used for the amino acid analysis.

The aim of this study was to characterise the collagen types in the lamina cribrosa and the trabecular meshwork and to determine whether 


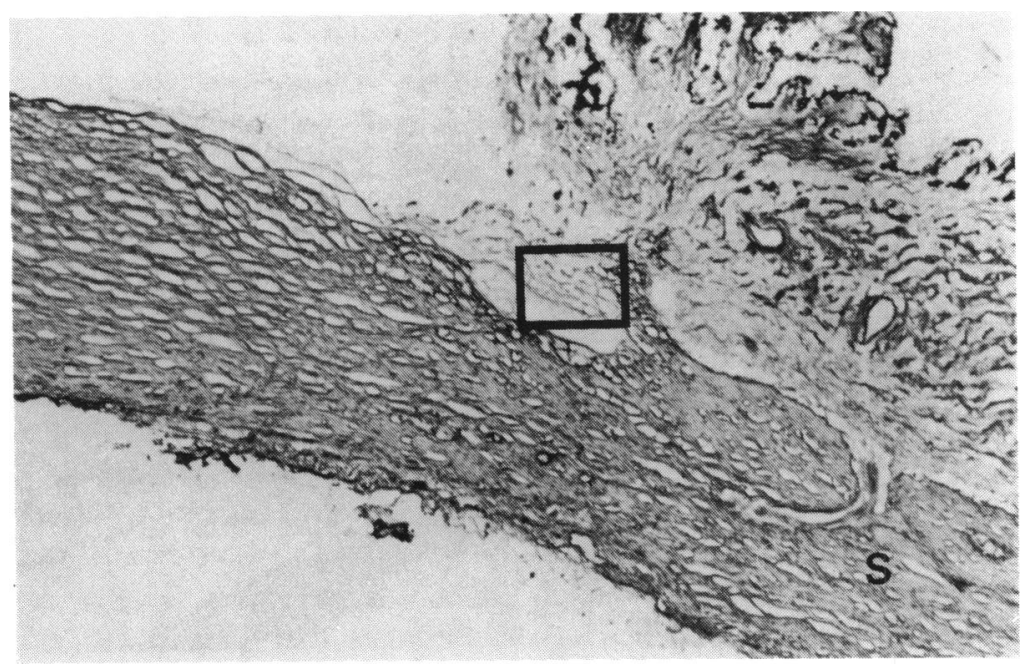

Fig. 1A

these structures have similar collagen compositions which differ from the collagen composition of the surrounding sclera in the normal human eye.

\section{Material and methods}

IMMUNOHISTOCHEMISTRY

Material. Ten normal eyes from persons aged 60 or more were obtained at necropsy, sectioned, and snapfrozen in liquid nitrogen $\left(-80^{\circ} \mathrm{C}\right)$ and stored at $-20^{\circ} \mathrm{C}$. Affinity-purified rabbit antibodies to bovine type I (calf skin) collagen, type III (human amnion) collagen, and type IV (human placenta) collagen were used. ${ }^{*}$

*The antibodies were kindly made available by Professor Furthmayr, Yale University, USA.
Fig. 1A-F A: Region of the trabecular meshwork. B: Insert. The trabecular meshwork (TM) is weakly stained by antibodies to type I (Fig. IA) but strongly stained with antibodies to type III (Figs. 1C, D) and type IV (Figs. IE, F), both showing staining of the peripheral portion of the trabecular beams. The sclera $(S)$ is stained with antibodies to type I collagen (Figs. 1A. B) but not type III (Figs. 1D, E) or type IV (Figs. IE, F) with the exception of vessels.

Methods. Sections 6-8 $\mu \mathrm{m}$ thick of the tissue samples were cut with a cryostat and dried on to glass slides. The sections were pretreated with cold acetone for $5 \mathrm{~min}$, incubated with phosphate buffered saline (PBS) for $5 \mathrm{~min}$, and incubated with rabbit antibodies to type I collagen 1:4, type III collagen 1:200, and type IV collagen 1:200 in a moist chamber for $30 \mathrm{~min}$ at room temperature. The

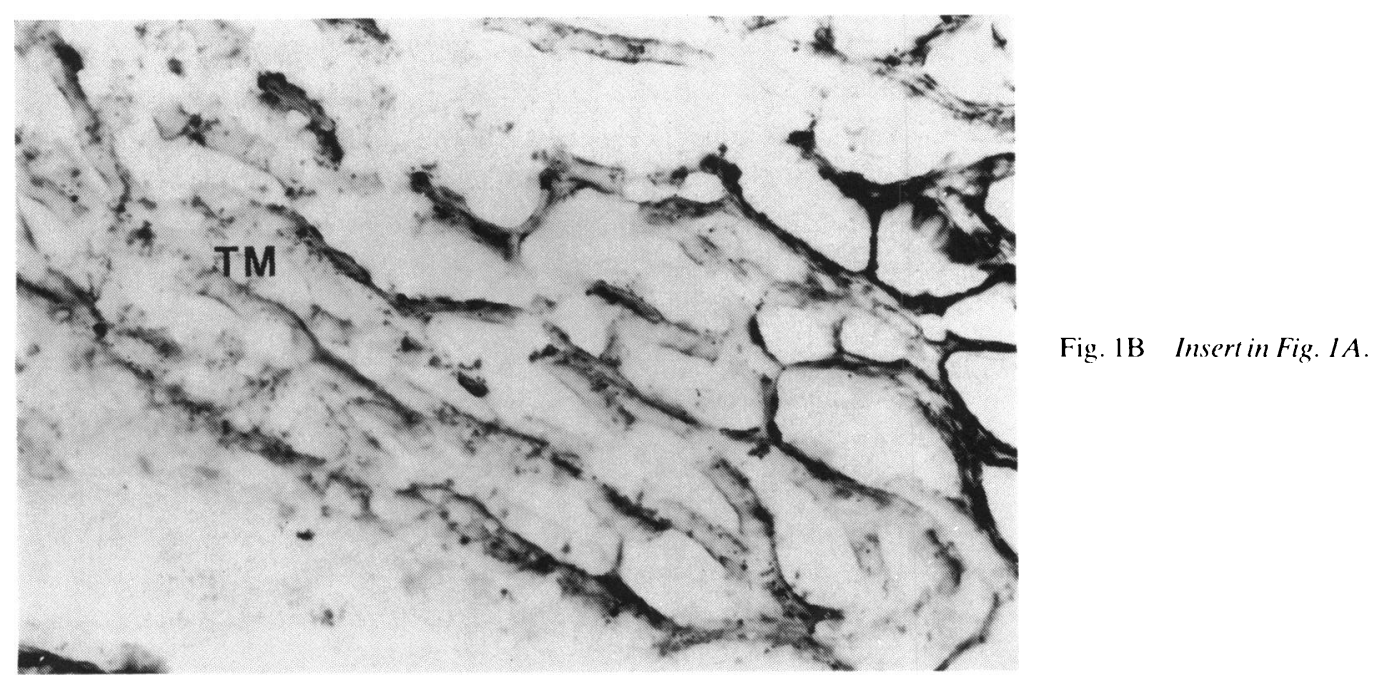


Fig. 1C

Fig. 1D Insert in Fig. IC.
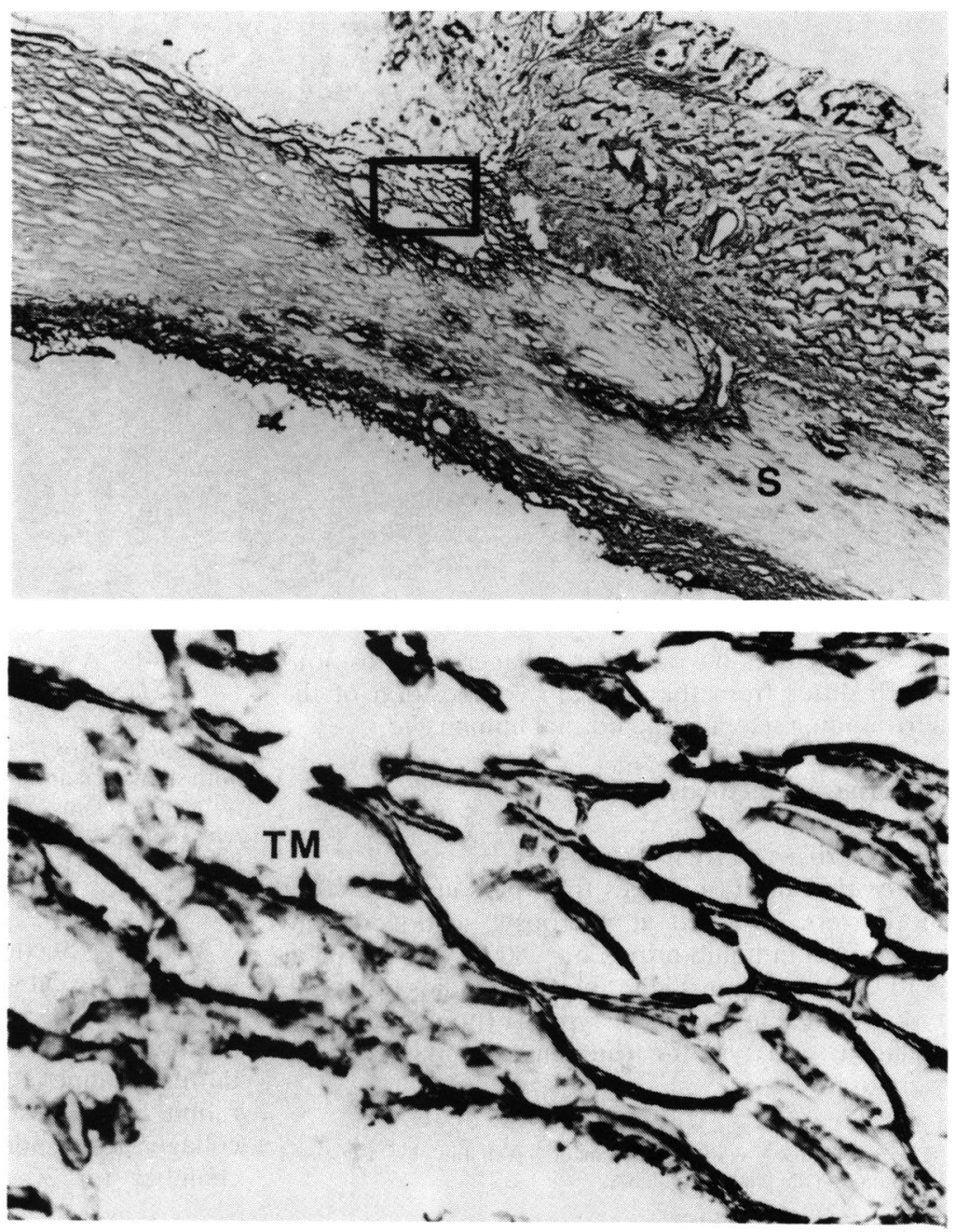

Fig. 1E

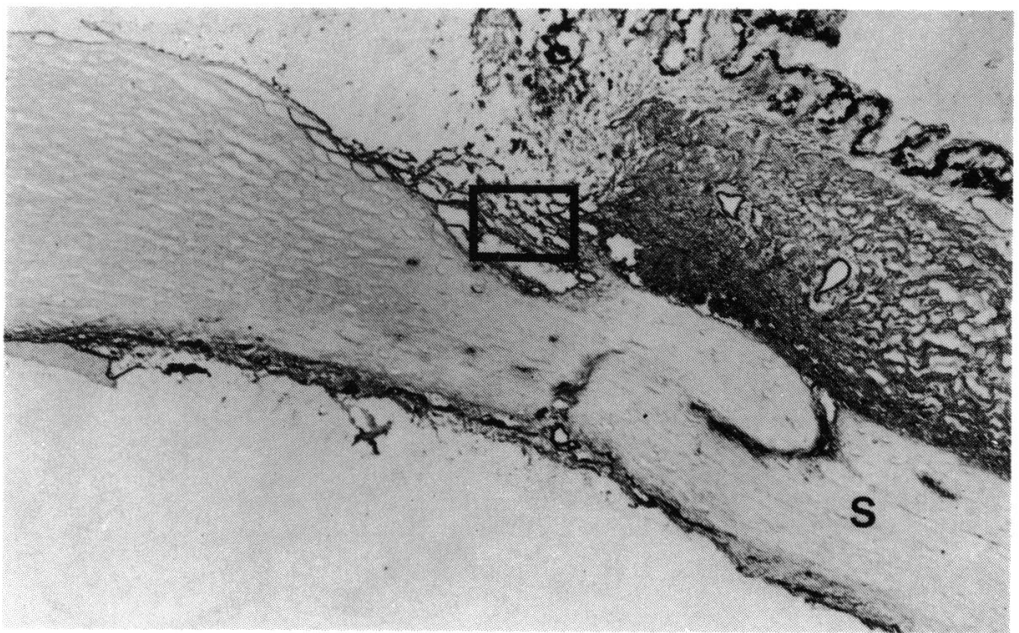




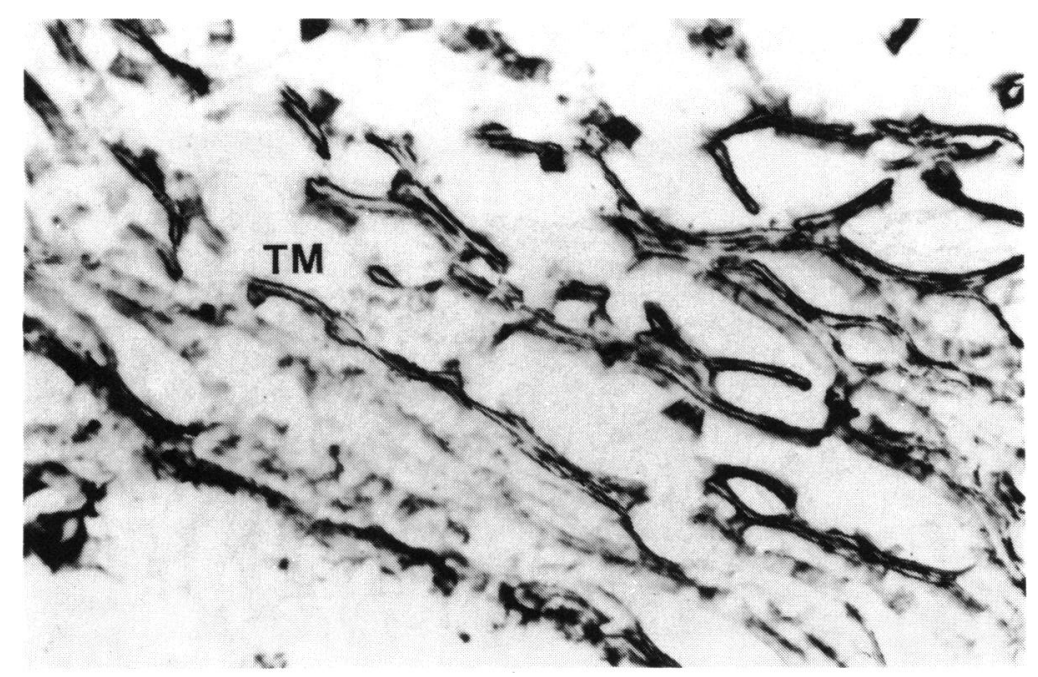

Fig. IF Insert in Fig. IE.

sections were then rinsed several times in PBS and subsequently stained with peroxidase-labelled antirabbit $\mathrm{IgG}$ and goat $\mathrm{IgG}$ for $30 \mathrm{~min}$, rinsed again several times in PBS, stained with diaminobenzidine for 3-5 min, and mounted in glycerol. As a control PBS buffer was used instead of primary antisera.

AMINO ACID ANALYSIS

Material. Four normal eyes taken at necropsy from

Fig. 2A-C Optic nerve head. The laminacribrosa is strongly stained with antibodies to type I (Fig. 2A), III (Fig. 2B), and IV (Fig. 2C). Types I and III show similar stromal staining, whereas type IV stains the basal membrane. The optic nerve $(\mathrm{ON})$ is strongly stained with antibodies to types I (Fig. 2A), III (Fig. 2B), and IV (Fig. 3C) in a similar fashion to the lamina cribrosa. two persons aged over 60 within 24 hours of death were used.

Methods. The eyes were dissected under a dissecting microscope and samples were taken from the trabecular meshwork, the sclera, and the lamina cribrosa as well as from the retrolaminar structures of the optic nerve.

The trabecular meshwork was excised with a fine pair of scissors in Schlemm's canal with extension along the Schwalbe line. The lamina cribrosa was dissected from the subarachnoid space to where it ends at the fusion of the pia and dura mater, the optic nerve being cut transversely with a razor blade and the lamina cribrosa excised from the sclera with a trephine.

The retrolaminar intrascleral part of the optic nerve was obtained, and a sample of scleral tissue

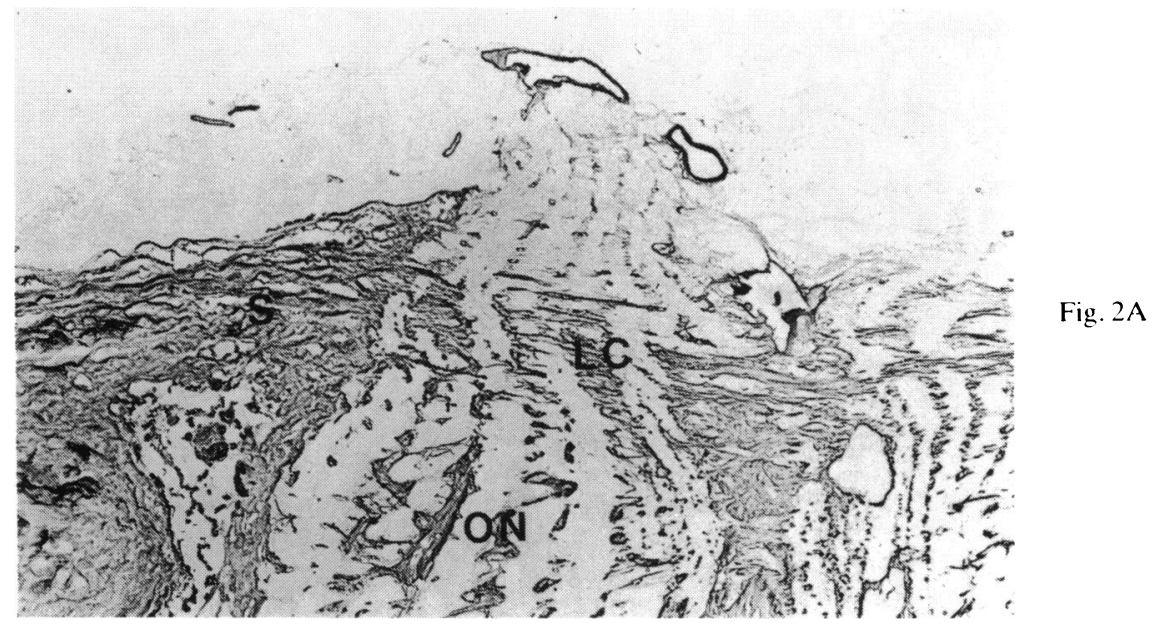


Fig. 2B

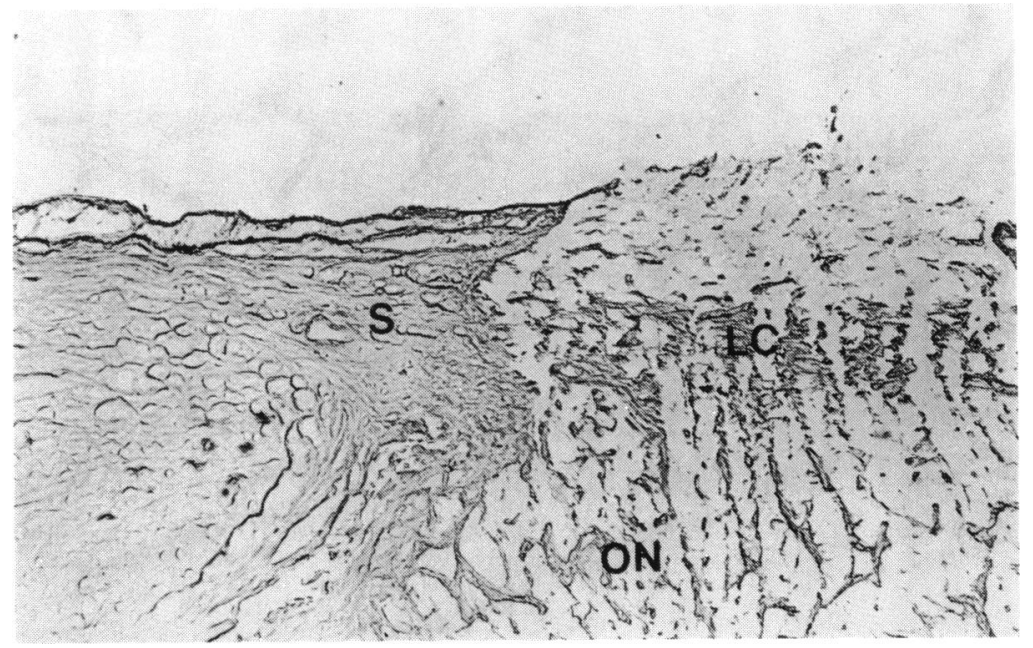

adjacent to the optic nerve was taken. The samples were stored in acetone. The collagen analysis was performed by measuring the concentrations ( $\mathrm{nmol} /$ mg defatted dried tissue) of Hyp, Hyl, and Pro in samples of trabecular meshwork $(T)$, lamina cribrosa (L), optic nerve $(\mathrm{O})$, and sclera $(\mathrm{S})$ as previously described.'

\section{Results}

\section{IMMUNOHISTOCHEMISTRY}

In the adult eye the immunohistochemical findings indicated the presence of types I, III, and IV collagen in both the trabecular meshwork and the lamina cribrosa (Figs. 1A-F, 2A-C). Types III and IV showed strong, consistent staining in both these tissues, whereas type I showed strong staining in the lamina cribrosa but somewhat weaker staining in the trabecular meshwork.

The sclera stained strongly for type I collagen but not for types III and IV. The optic nerve stained for types I, III, and IV collagen.

Fig. 3 shows the distribution of collagen types in a schematic eye.

AMINO ACID ANALYSIS

The mean range of the concentrations of Hyp. Hyl, and Pro (nmol/mg defatted dried tissue) were recorded (Table 1 ), and the molar ratio of Hyp to Hyl was calculated (Table 2 and Fig. 4). In all four eyes the highest concentration of Hyp, Hyl, and Pro was found in the sclera and the lowest concentration in the lamina cribrosa. The Hyl concentration was almost equal in the sclera and the trabecular mesh-

Fig. 2C

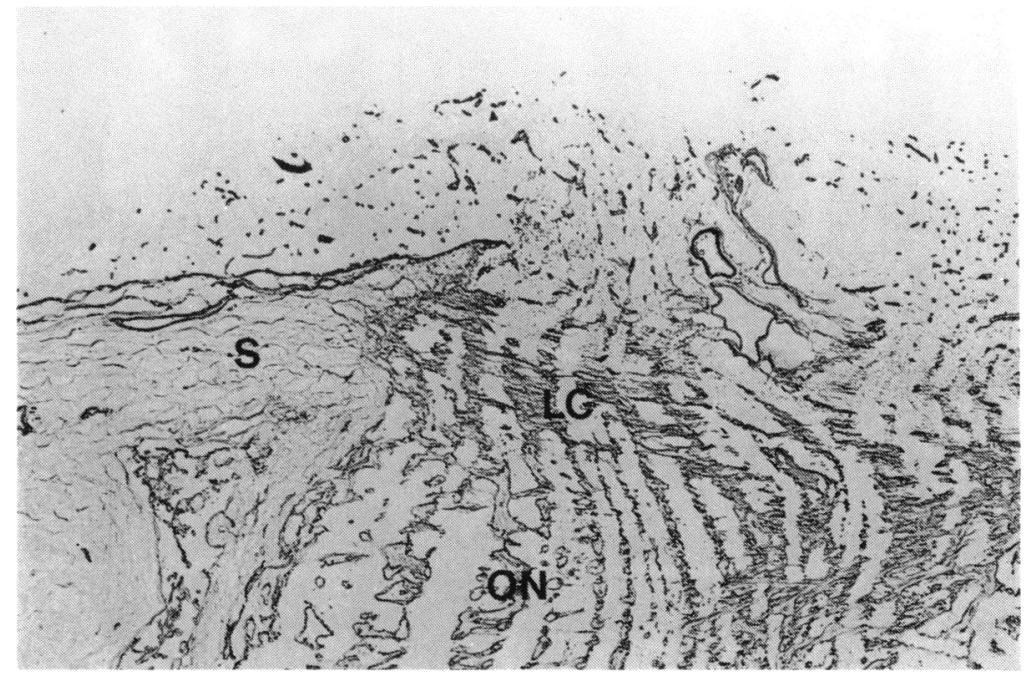




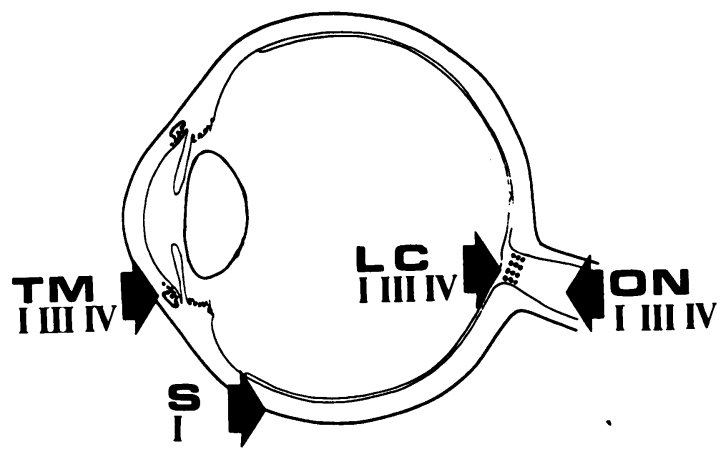

Fig. 3 The collagen types I, III, and IV were found in the trabecular meshwork (TM), lamina cribrosa (LC), and the optic nerve (ON). Only type I was seen in sclera (S).

Table 1 Concentrations of Hyp, $H y l$, and Pro (nmolling defatted dried tissue) are given as mean (and range)

\begin{tabular}{llll}
\hline & $H_{y p}$ & $H_{y} l$ & Pro \\
\hline Trabecular meshwork & $390(3(0)-50(0))$ & $37(26-49)$ & $610(540-690)$ \\
Lamina cribrosa & $230(180-270)$ & $21(14-27)$ & $470(40(0)-52(0)$ \\
Optic nerve & $340(3(0)-390)$ & $25(20)-28)$ & $515(440-580)$ \\
Sclera & $610(590-650)$ & $44(37-50)$ & $850(820-870)$ \\
\hline
\end{tabular}

Table 2 Hyp/Hyl ratios

\begin{tabular}{rllll}
\hline & $\begin{array}{c}\text { Lamina } \\
\text { cribrosa }\end{array}$ & $\begin{array}{l}\text { Trabecular } \\
\text { meshwork }\end{array}$ & $\begin{array}{l}\text { Optic } \\
\text { nerve }\end{array}$ & Sclera \\
\hline Sample cyes I a & 13.1 & 11.4 & 14.7 & 14.8 \\
b & 11.7 & 13.7 & 15.4 & 16.1 \\
II a & 9.4 & 9.6 & 11.6 & 11.9 \\
b & 9.4 & 8.4 & 1.3 .3 & 12.6 \\
\hline
\end{tabular}

Four eyes from two persons. I and II.

work, though the Hyp concentration was about $50 \%$ higher in the sclera. The mean Hyp to Hyl ratio was 13.8 in the sclera and 13.8 in the optic nerve, which was significantly higher than in the two other tissues. The ratio of Hyp to Hyl was equal to that in the lamina cribrosa (10.9) and the trabecular meshwork $(10 \cdot 8)$. Fig. 4 shows the distribution of the individual results.

\section{Discussion}

The immunohistochemical findings showed the presence of collagen types I, III, and IV in the lamina cribrosa and in the trabecular meshwork. However, the staining of the type I collagen in the trabecular meshwork was weak. In a previous similar study ${ }^{2}$ only type IV was detected in the trabecular meshwork. However, biochemical evidence" suggested that type $\mathrm{I}$ is also present, as amino acid ratios similar to those of the dermis, which is rich in type I collagen, were obtained for the trabecular meshwork. The collagen

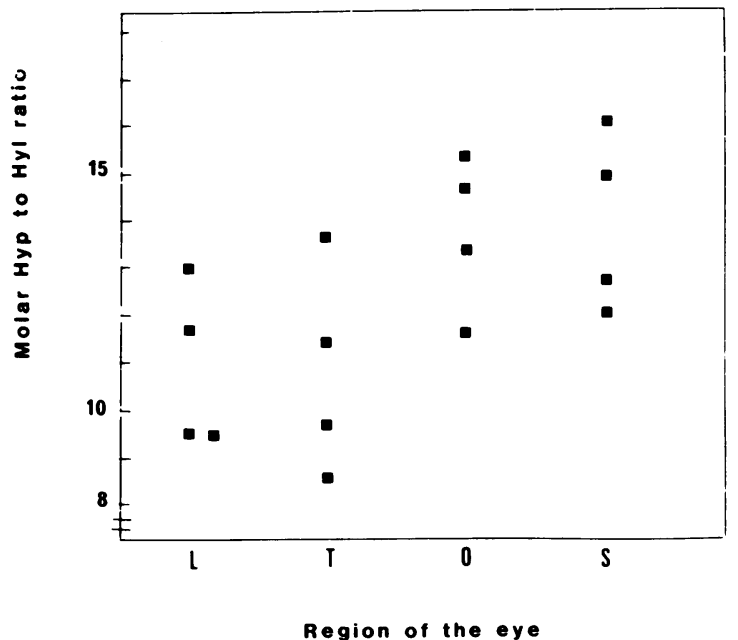

Fig. 4 The molar radio of hydroxyproline ( $\mathrm{Hyp}$ ) to hydroxylysine $(\mathrm{Hyl})$ was determined in samples of lamina cribrosa (L), trabecular meshwork $(\mathrm{T})$, optic nerve $(\mathrm{O})$, and sclera (S) from four eyes.

type distribution found in the lamina cribrosa is in agreement with earlier immunohistochemical work by Konomi et al. ${ }^{+}$

The Hyp to Hyl ratio of a tissue depends on the relative distribution of the molecular types of collagen, on the degree of cross linking, and on possible defects in the amino acid sequence. A relatively increased content of basement membranes causes a decrease in the Hyp to Hyl ratio because of a high content of $\mathrm{Hyl}$ in type IV collagen.5 This may. explain the low Hyp to Hyl ratio and relatively high concentrations of $\mathrm{Hyl}$ in the lamina cribrosa and the trabecular meshwork as compared with the sclera and the optic nerve.

The similarity of the collagen type distribution between the trabecular meshwork and the lamina cribrosa suggested by the immunohistochemical findings is in agreement with the similarity in the Hyp to Hyl ratio, which indicates a resemblance in collagen composition of these structures. The results of the present study reveal the similarities between structures of particular interest in chronic open angle glaucoma. A common change of the supporting elements in these tissues, as suggested in earlier reports, ' is of interest and will encourage further studies of the mechanical properties of collagen as well as a more detailed analysis of the collagen types present in normal and glaucomatous eyes.

The authors thank Mr Ole Christensen. Mrs Berith Spăngberg. and Mrs Margareta Oskarsson for skilfull technical assistance, and $\mathrm{Dr}$ Peter Biberfeld for valuable advice. The study has been sponsored by the Crownprincess Margareta's Working Fund. 


\section{References}

1 Tengroth B. Ammitzboll T. Changes in the content and the composition of collagen in the glaucomatous eye: hasis for a new hypothesis in the genesis of chronic open angle glaucoma-a preliminary report. Acta Ophthalmol (Kbh) 1984; 62: 99-108.

2 Rodrigues MM, Katz SI, Foidart I-M. Spaeth GL. Collagen, factor VIII and immunoglobulins in the human aqueous drainage channels. Ophthalmology 198(): 87: 337-44.
3 Horstmann H-J. Rohen JW. Sames K. Age-related changes in the composition of proteins in the trabecular meshwork of the human cyc. Mech Ageing Dev 1983; 21: 212-36.

4 Konomi M. Hayaski T, Sano J, et al. Immunohistochemical localization of type I. III and IV in the sclera and choroid of bovine, rat and normal and pathological human eyes. Biomed Res 1983: 4: 451-8.

5 Miller EJ. Gay S. Collagen: an overview. Methods in Enzymol 1982; 82A: 3-32. 